\title{
CÁlCULO dE LA PÉRDIDA DE TRANSMISIÓN ACÚSTICA Y DE CONTRAPRESIÓN EN SILENCIADORES, MEDIANTE EL MÉTODO DE LOS ELEMENTOS FINITOS
}

\author{
Calculation of Transmission loss and Back Pressure in mufflers, \\ by means of Finite Element Method
}

\author{
Miguel Ángel Méndez López ${ }^{*}$ \\ Mario Alejandro Avella Suarez** \\ Juan Felipe Giraldo Ávila*** \\ Jhon Freddy Ochoa Avendaño****
}

Recibido: 14 de agosto de 2017

Aceptado: 11 de septiembre de 2017

\section{Resumen}

En este artículo se presenta la simulación de la pérdida de transmisión acústica y la contrapresión de silenciadores vehiculares por medio del método de los elementos finitos. Las dos variables mencionadas son inversamente proporcionales entre sí, razón por la que se busca su equilibrio en el diseño de silenciadores, para lograr que la pérdida de transmisión acústica sea alta y la caída de presión sea baja. Los cálculos fueron obtenidos mediante el uso de los programas informáticos ANSYS APDL y Siemens NX Flow. Los resultados de las simulaciones fueron comparados y validados con los experimentos y las soluciones numéricas presentados por otros autores.

Palabras clave: método elementos finitos, atenuación sonora, pérdida de transmisión acústica, caída de presión.

\section{Abstract}

This article presents the simulation of Transmission Loss and the Back Pressure of mufflers by means of Finite Element Method. These two variables are inversely proportional, reason why its balance is searched in muffler's design process to achieve a high Transmission Loss and a low Back Pressure. The calculations were obtained using the Ansys APDL and Siemens NX Flow software. The simulations of the results obtained were compared and validated with the experimental results and the sub-structured modal method presented by other authors.

Keywords: finite element method, sound attenuation, acoustic transmission loss, pressure drop.

\footnotetext{
* Estudiante de Ingeniería Mecánica. Grupo de investigación Diseño Avanzado, línea de investigación Modelado Computacional, Fundación Universidad de América, Bogotá, Colombia.miguel.mendez@estudiantes.uamerica.edu.co

** Estudiante de Ingeniería Mecánica. Grupo de investigación Diseño Avanzado, línea de investigación Modelado Computacional, Fundación Universidad de América, Bogotá, Colombia. mario.avella@ estudiantes.uamerica.edu.co ${ }^{\star * \star}$ Ingeniero mecánico, magíster en Ingeniería Mecánica, Universidad Nacional de Colombia. Docente Investigador, Departamento de Ingeniería Mecánica, grupo de investigación Grupo Diseño Avanzado, línea de investigación Modelado Computacional, Fundación Universidad de América, Bogotá, Colombia. ORCID:http://orcid.org/0000-0002-19022555. juan.girlado@profesores.uamerica.edu.co

${ }_{* * * *}$ Ingeniero mecánico, magíster en Ingeniería Materiales y Procesos, Universidad Nacional de Colombia. Docente Investigador, Departamento de Ingeniería Mecánica, grupo de investigación Diseño Avanzado, línea de investigación Modelado Computacional Fundación Universidad de América, Bogotá, Colombia. ORCID

https://orcid.org/0000-0002-7262-6200. jhon.ochoa@profesores.uamerica.edu.co
} 


\section{INTRODUCCIÓN}

Los silenciadores son comúnmente usados en una gran cantidad de aplicaciones; una de las más importantes es la atenuación del ruido en vehículos propulsados por motores de combustión interna. Las leyes ambientales vigentes que rigen la contaminación auditiva (ver Resolución 0627 de 2006) demandan que los silenciadores sean diseñados con una alta calidad y rendimiento y que cumplan con las normas vigentes que regulan el límite máximo de generación de ruido.

El desempeño de un silenciador está determinado por dos factores: a) nivel de atenuación sonora, que indica la cantidad de ruido reducido por el silenciador en decibeles (dB) para un rango de frecuencias específicas (Pal, 2015), y b) caída de presión, ${ }^{1}$ definida como la diferencia de presión que necesita el fluido de escape para superar la resistencia hidráulica generada por el silenciador y así facilitar el flujo de los gases de escape hacia el ambiente (Vergara, 2015). De manera que se debe buscar un silenciador que atenúe niveles altos de presión sonora (ruido) y genere bajos niveles de caída de presión.

En el campo de la modelado computacional y simulación, se han utilizado principalmente tres técnicas para el cálculo aproximado del nivel de atenuación sonora: el método de los elementos finitos (MEF), el método de la matriz de transferencia (MMT) y el método de los tres puntos (MTP). El MEF se basa en la solución numérica de la ecuación diferencial de Helmholtz (Yasuda, Wu, Nakagawa y Nagamura, 2013), en la que se realiza las tres etapas típicas del método, a saber, formulación variacional, discretización y solución numérica del sistema matricial que resulta de la formulación (García, 2014). Por su parte, el MMT, también conocido como método de los cuatro polos, basa el cálculo de la atenuación de ruido en la medición de cuatro variables principales del ducto estudiado: presión a la entrada, velocidad a la entrada, presión a la salida y velocidad a la salida. Estas variables generalmente deben ser medidas de forma experimental mediante instrumentación específica para cada caso. E1 MMT establece relaciones algebraicas entre esas cuatro variables, relaciones que son planteadas matemáticamente en una matriz que recibe el nombre de matriz de transferencia (McMahon, 2014); esta matriz genera un sistema de ecuaciones que deben ser resueltas para conocer los valores del nivel de atenuación de ruido y la caída de presión (Mori, 2016). Finalmente, el MTP funciona de manera similar al MMT; sin embargo, en este caso, el cálculo se realiza basándose en la medición de tres parámetros y no en cuatro, como en el caso del MMT. Los parámetros medidos corresponden a la presión en tres puntos específicos del ducto estudiado, donde la atenuación de ruido queda determinada a partir de un análisis matemático de las presiones de las ondas entrantes y su transmisión (Cui y Huang, 2016). Este análisis da como resultado un parámetro conocido como pérdida de transmisión acústica (que en adelante se denominará TL, sigla de transmission loss), que en este trabajo se utilizó para el análisis de desempeño de silenciadores.

El estudio de estos dos factores se basó en un análisis fundamentado en simulaciones computacionales llevadas a cabo en los programas informáticos ANSYS APDL, para TL, y NX Flow, para la pérdida de presión. Ambos programas permiten obtener soluciones aproximadas de ecuaciones diferenciales mediante el método de los elementos finitos (MEF), y proporcionan las condiciones y el entorno adecuados para el estudio en cuestión.

Por su parte, APDL es una herramienta de programación utilizada en el programa ANSYS, que permite el desarrollo del análisis acústico usando listas de instrucciones parametrizadas.

De manera similar, NX Flow es una implementación informática de la dinámica de fluidos computacional (CFD), que proporciona herramientas para modelar y simular el comportamiento

\footnotetext{
${ }^{1}$ Para el caso de sistemas de escape, la caída de presión es más conocida como contrapresión.
} 
de los fluidos dentro de piezas y ensamblajes complejos (Siemens, 2013), en este caso, los gases de escape en el interior de los silenciadores.

En este artículo se analiza la efectividad en la atenuación de ruido de diferentes tipos de silenciadores, mediante el cálculo de la pérdida de transmisión acústica por medio del programa ANSYS. Los resultados obtenidos son comparados con los resultados experimentales y numéricos reportados por Torres (2006), los cuales fueron generados mediante el método modal subestructurado, que es una variación del MMT.

\section{Materiales y métodos}

\section{Definiciones teóricas iniciales}

\section{Silenciadores reactivos}

Son utilizados para reducir el ruido generado por la combustión del motor. Estos silenciadores funcionan con el principio de interferencia destructiva, es decir, las ondas se reflejan y se cancelan con una de la misma amplitud. Existen dos tipos de silenciadores reactivos: cámara simple y ducto perforado (Pal, 2015).

\section{Impedancia}

Se presenta cuando la onda acústica cambia de un medio de propagación a otro. Físicamente, es la pérdida de energía de la onda como consecuencia de las propiedades absorbentes de la frontera, pues al cambiar de medio de propagación, hay una reflexión y refracción de la onda. De manera que la impedancia es una medida cuantitativa que establece cómo un cambio de medio de propagación puede atenuar o amortiguar la propagación de onda (García, 2014).

\section{Pérdida de transmisión acústica (TL)}

Índice de atenuación sonora que calcula la caída de presión acústica de una onda sonora que se propaga entre dos puntos de un sistema de escape. Este valor no depende de la fuente de ruido ni de la impedancia de la terminación del silenciador (Martínez, 2010).

\section{Contrapresión}

Diferencia entre la presión de escape media y la presión ambiente a la salida. Es la presión opuesta que ejerce el fluido a salir del sistema de escape (Selamet, Denia y Besa, 2003).

\section{Porosidad}

Porcentaje del volumen total del ducto interno en el silenciador que se va remplazar por perforaciones. Para un $100 \%$ del ducto, la porosidad de este artículo varía entre el 10 y $11.3 \%$.

\section{Metodología}

El análisis del desempeño de un silenciador reactivo se realizó a través del estudio exhaustivo de las propiedades físicas del fluido de escape (presión, velocidad y temperatura), así como se propone en el estudio de Mishra, Kar, Mishra y Gupta (2016).

\section{Propiedades físicas del fluido de estudio}

Las propiedades de trabajo del fluido de escape en un silenciador en condiciones de trabajo son: 
Velocidad del fluido: $2.45 \frac{\mathrm{m}}{\mathrm{s}}$

Temperatura de trabajo: $363^{\circ} \mathrm{C}$

Densidad del fluido (aire): $0.5555 \frac{\mathrm{Kg}}{\mathrm{m}^{3}}$

\section{Modelo acústico}

Las propiedades de la onda asumidas en este estudio son velocidad $(v=343.24 \mathrm{~m} / \mathrm{s}$ ) y presión incidente $\left(p_{i}=a t m\right)$. Los casos a estudiar son tomados de la tesis doctoral de Torres (2006) y se identifican con las siguientes abreviaturas:

Tabla 1. Silenciadores evaluados

\begin{tabular}{llc}
\hline Abreviatura & \multicolumn{1}{c}{ Tipo de silenciador } & Porosidad \\
\hline SCP & Silenciador concéntrico perforado & $10 \%$ \\
SEP & Silenciador excéntrico perforado con porosidad & $11.3 \%$ \\
\hline
\end{tabular}

En este estudio se simula el índice de TL, el cual evalúa la diferencia entre los niveles de presión acústica de las ondas incidentes y trasmitidas, método que resulta ser el más efectivo para este tipo de aplicaciones (Martínez, 2010). La medición para el cálculo de TL se toma como se muestra en la figura 1.

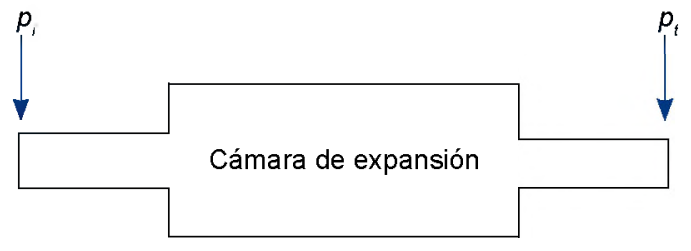

Figura 1. Puntos de medición para un silenciador.

Fuente: Méndez y Avella (2017).

$$
T L=20 \log 10\left|\frac{p_{i}}{p_{t}}\right|
$$

Donde $p_{i}$ es el punto de presión incidente y $p_{t}$ es el punto de presión transmitida.

La solución de la ecuación 1 genera los resultados de atenuación sonora en un rango de frecuencias, razón por la que se acopla con la ecuación de onda de Helmholtz, que a su vez describe la propagación de onda sonora en ductos (como es el caso del sistema de escape). El resultado del acople queda expresado como muestra la ecuación 2 (Nefske, Wolf, y Howell, 1982)

$$
\bar{V}^{2} p+k^{2} p=0
$$

Donde $\bar{V}^{2} p$ corresponde al Laplaciano de la presión, el cual representa la propagación de las ondas en el fluido, y $k$ es el número de onda y es igual a $\omega / \mathrm{c}(\omega:$ la frecuencia angular; c: velocidad del sonido). Adicionalmente, la ecuación 2 hace parte de la formulación de las condiciones de frontera presentes en los silenciadores reactivos (ver figura 2). 


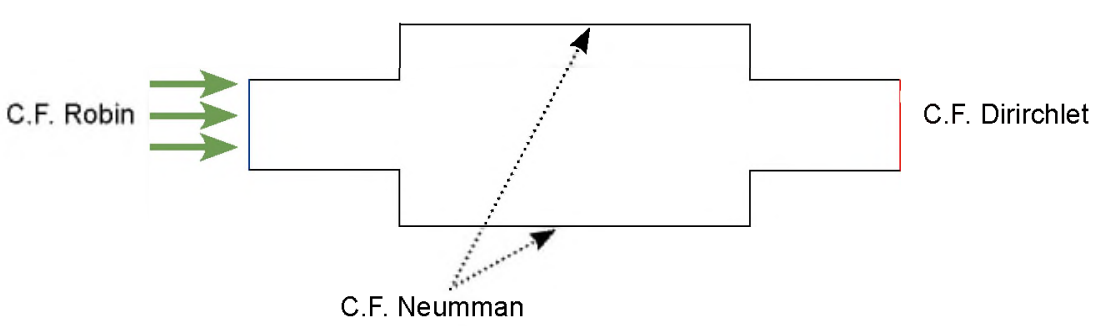

Figura 2. Condiciones de frontera para un silenciador de cámara simple.

Fuente: Méndez y Avella (2017).

La línea de color rojo en la figura 2 representa un límite de radiación que simula la salida al ambiente, donde no hay reflexión de onda al haber una propagación del $100 \%$ fuera del sistema. Esta es una condición de frontera tipo Dirichlet ${ }^{2}$, la cual queda expresada en la ecuación 3.

$$
p\rceil_{\partial \Omega}=0
$$

Donde $p$ es presión sonora y $\partial \Omega$ es la frontera.

La frontera de color negro en la figura 2 representa una condición de aislamiento acústico. En estos bordes no hay absorción de onda ni trasmisión al exterior, es decir, la onda sonora se refleja y se cancela con una onda de la misma amplitud. Esta es una condición de frontera tipo Neumman ${ }^{3}$, y se expresa de la siguiente manera:

$$
\left.\frac{\partial p}{\partial \vec{n}}\right\rceil_{\partial \Omega}=0
$$

Donde $\vec{n}$ es el vector normal a la frontera.

La línea de color azul en la figura 2 representa una combinación lineal de la presión y su variación en la frontera. Esta condición acústica describe un cambio de medio (cambio de impedancia), que se refiere a cómo un nuevo medio (cambio de sección de área) puede atenuar la propagación de onda. Esta condición de frontera es denominada de tipo Robin y queda expresada de la siguiente manera:

$$
Z=\frac{p}{U} \cdot\left[\frac{P a^{*} s^{-1}}{m}\right]
$$

Donde $p$ es Presión sonora y $U$ es Velocidad de la partícula.

\section{Caída de presión}

La caída de presión se da como consecuencia de las discontinuidades en la geometría del sistema de escape. Este artículo estudia el silenciador de un sistema de escape convencional, razón por

\footnotetext{
${ }^{2}$ La condición de frontera Dirichlet consiste en dar un valor fijo de la variable dependiente en los puntos del contorno deseados.

${ }^{3}$ La condición de frontera Neumman representa la derivada normal de la condición de frontera Dirichlet respecto a la variable independiente.
} 
la que la caída de presión obedece a la presencia de componentes perforados y cambios de sección transversal a lo largo de la geometría del silenciador. El cálculo se realiza por medio de la ecuación de conservación de momento de Navier-Stokes:

$$
\varrho_{\infty}\left[\frac{\partial v}{\partial t}+(v \cdot \nabla) v\right]=-\nabla p+\eta \nabla^{2} v+\varrho_{\infty} g
$$

Donde:

$\varrho_{\infty}\left[\frac{\partial v}{\partial t}+(v \cdot \nabla) v\right]=$ fuerzas de inercia

$-\nabla p=$ fuerzas de presión

$\eta \nabla^{2} v=$ fuerzas viscosas

$\varrho_{\infty}=$ fuerzas externas aplicadas al fluido

$b$ = gravedad

\section{Modelo computacional acústico}

\section{Parámetros de entrada}

El programa ANSYS tiene un módulo para la solución de problemas acústicos. Utiliza la ecuación de Helmholtz como base para el estudio de silenciadores, con la que es posible realizar el análisis de vibraciones forzadas en diferentes cavidades tridimensionales.

Las simulaciones fueron realizadas a temperatura ambiente $\left(20^{\circ} \mathrm{C}\right)$, con el fin de validar los resultados a partir de los resultados presentados por Torres (2006); los demás parámetros de entrada se presentan en la tabla 2. Es importante mencionar en este punto que las unidades de cada variable corresponden al sistema internacional de unidades.

Tabla 2. Parámetros de entrada simulación acústica

\begin{tabular}{lcc}
\hline \multicolumn{1}{c}{ Variable } & Expresión & Valor \\
\hline Densidad del aire & $\rho$ & 1.2054 \\
Velocidad del sonido & $C_{0}$ & 343.24 \\
Velocidad de la masa & $z_{0}=\rho^{*} c_{0}$ & - \\
Frecuencia de trabajo máxima & freq $q_{E}$ & 3500 \\
Longitud de onda para la frecuencia máxima & wave $=\frac{C_{0}}{f r e q_{E}}$ & - \\
Presión exterior & $\rho$ & 1 \\
Excitación de velocidad normal & $v_{n}=\frac{p}{\left(\rho * C_{0}\right)}$ \\
\hline
\end{tabular}

\section{Configuración geométrica}

La configuración geométrica del silenciador se obtuvo mediante el módulo de creación de geometrías del programa ANSYS APDL. Los parámetros geométricos se presentan en la tabla 3. La geometría resultante se muestra en la figura 3. 
Tabla 3. Parámetros geométricos silenciador

\begin{tabular}{lcc}
\hline \multicolumn{1}{c}{ Parámetro } & Expresión & Valor \\
\hline Radio del ducto de entrada & $r_{\text {ent }}$ & 0.0268 \\
Longitud del ducto de entrada & $J_{\text {ent }}$ & 0.1 \\
Radio del ducto de salida & $r_{\text {sat }}$ & 0.0268 \\
Longitud del ducto de salida & $I_{\text {sat }}$ & 0.1 \\
Longitud de la cámara de expansión & $I_{c h a b}$ & 0.148 \\
Radio cámara de expansión & $r_{\text {chamb }}$ & 0.091 \\
\hline
\end{tabular}

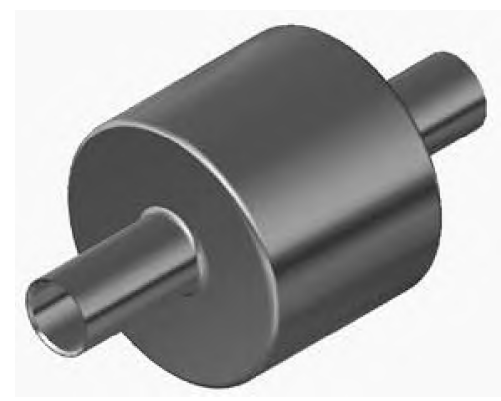

Figura 3. Geometría en 3D.

Fuente: Méndez y Avella (2017).

\section{Tipo de elemento y enmallado}

El elemento utilizado en la simulación corresponde al fuid30, que es incluido por ANSYS APDL para la solución de problemas de flujo medio e interacción fluido estructura. También es ampliamente utilizado en aplicaciones que incluyen la propagación de ondas sonoras. Por otra parte, este elemento permite una discretización que toma en cuenta el acople entre la presión acústica y el movimiento estructural en la interfaz (Ansys INC., 2017). El enmallado se realiza de forma tradicional sin ningún tipo de refinamiento; se aplican las condiciones de frontera Dirchlet y Neumman como se muestra en la figura 2.

\section{Modelo computacional para la caída de presión}

La simulación de la caída de presión se llevó a cabo con el programa NX Flow. Los datos obtenidos fueron validados mediante un análisis de convergencia de malla (Méndez y Avella, 2017), análisis que proporciona un criterio de aproximación de resultados convergentes.

La caída de presión se interpreta de manera simplificada como la diferencia entre la presión estática de entrada $\left(P_{\text {in }}\right)$ y la presión estática de salida $\left(P_{\text {out }}\right)$, como se muestra en la ecuación $7 . \mathrm{E} 1$ valor de $P_{\text {in }}$ se calculó en el programa NX Flow a partir de las condiciones iniciales de velocidad de entrada $\left(V_{\text {in }}=2.45 \mathrm{~m} / \mathrm{s}\right)$ y presión de salida: $\left(P_{\text {out }}=1 \mathrm{~atm}\right)$.

$$
\Delta P=P_{\text {in }}-P_{\text {out }}(P a)
$$




\section{Resultados}

Al ejecutar el código en APDL, se obtiene la gráfica de la pérdida de transmisión acústica para los silenciadores SCP (figura 4) y SEP (figura 5).

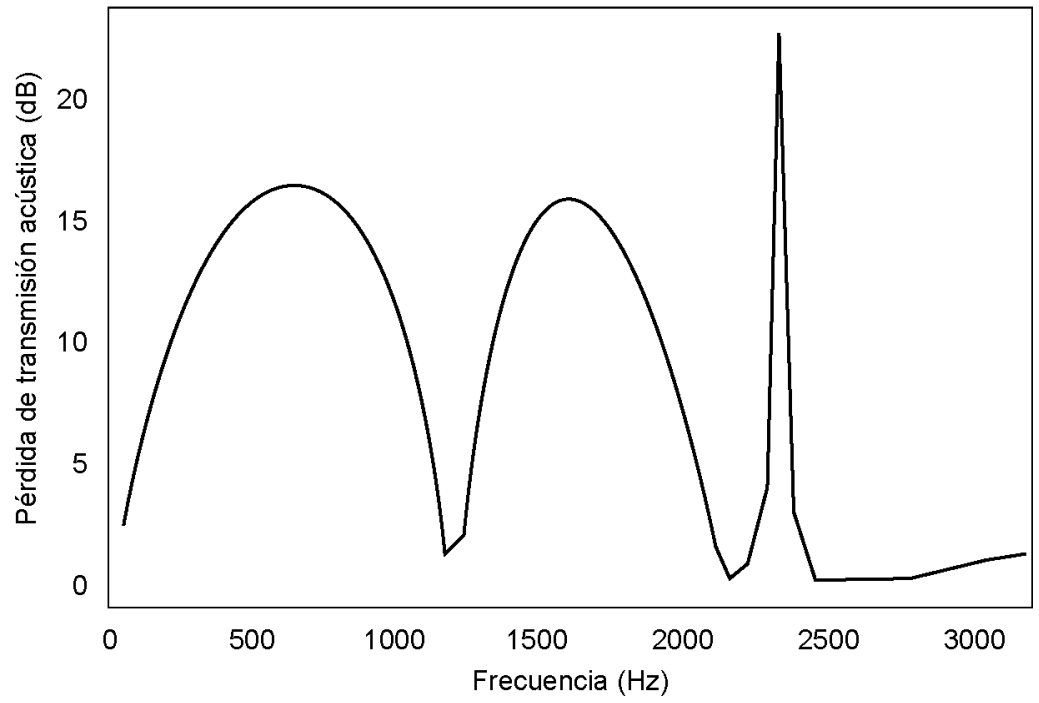

Figura 4. Gráfica pérdida de transmisión acústica TL para el SCP.

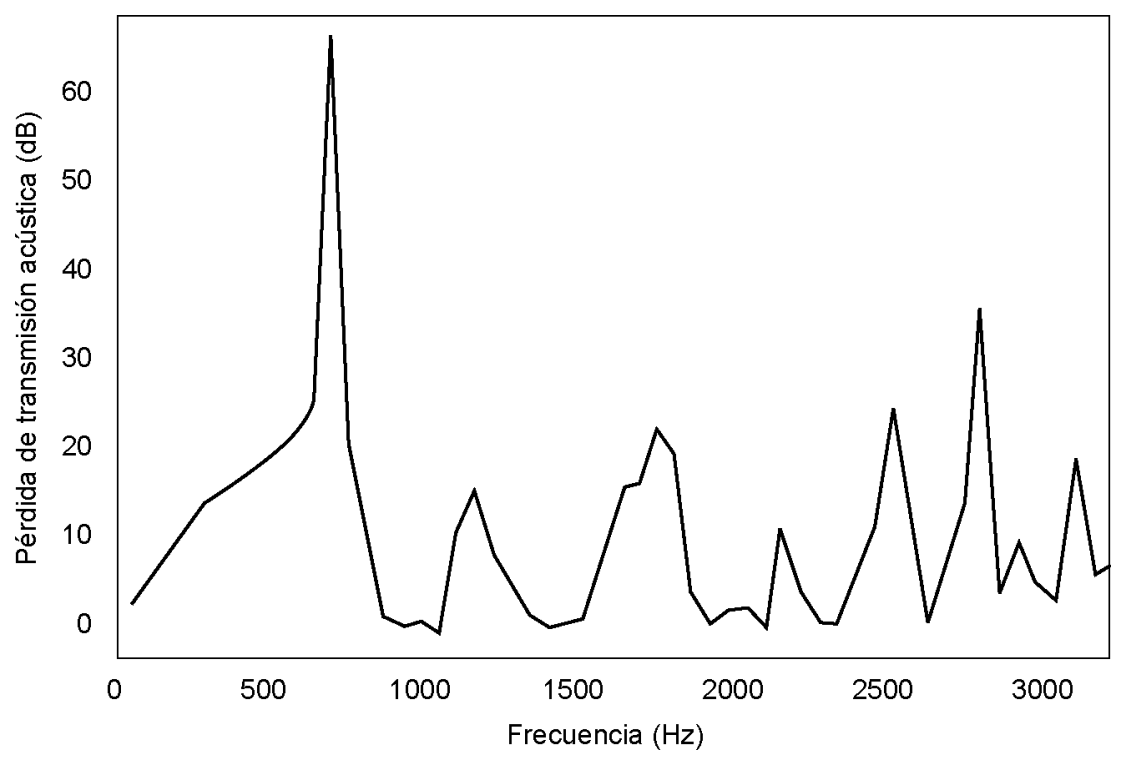

Figura 5. Gráfica pérdida de transmisión acústica TL para el SEP.

Se presentan los niveles de atenuación sonora en $\mathrm{dB}$ para el rango de frecuencias, que comprende desde los $0 \mathrm{~Hz}$ hasta los $3500 \mathrm{~Hz}$ (frecuencias bajas). Los resultados obtenidos para la pérdida de transmisión acústica a diferentes frecuencias deben interpretarse bajo la consideración de que a mayor amplitud de la onda, mayor es la cantidad de ruido atenuado. De esta forma es posible evidenciar en la figura 4 que el intervalo de atenuación para el SCP va desde los $15 \mathrm{~dB}$ hasta los $24 \mathrm{~dB}$, prin- 
cipalmente para las frecuencias que varían entre 0 y $2400 \mathrm{~Hz}$. La figura 5, que corresponde al SEP, muestra que los niveles de atenuación son más altos, desde los $15 \mathrm{~dB}$ hasta los $75 \mathrm{~dB}$. Adicionalmente, para el SEP se presenta una oscilación en los diferentes rangos de frecuencias.

En las figuras 6 y 7 se realizó la comparación del método utilizado en este artículo con el método modal subestructurado y los resultados experimentales presentados por Torres (2006). Tanto para el caso del SCP (ver figura 6), como para el caso del SEP (ver figura 7), es posible observar una buena aproximación entre los resultados obtenidos en este artículo y los resultados experimentales obtenidos por Torres (2006). En el caso específico del SCP, se evidencia que con el método de los elementos finitos se logra una mejor aproximación a los resultados experimentales que con el método modal subestructurado. Adicionalmente, en el caso del SEP, el método utilizado en esta investigación logra aproximaciones cercanas en ciertos rangos donde el método subestructurado no lo logra.

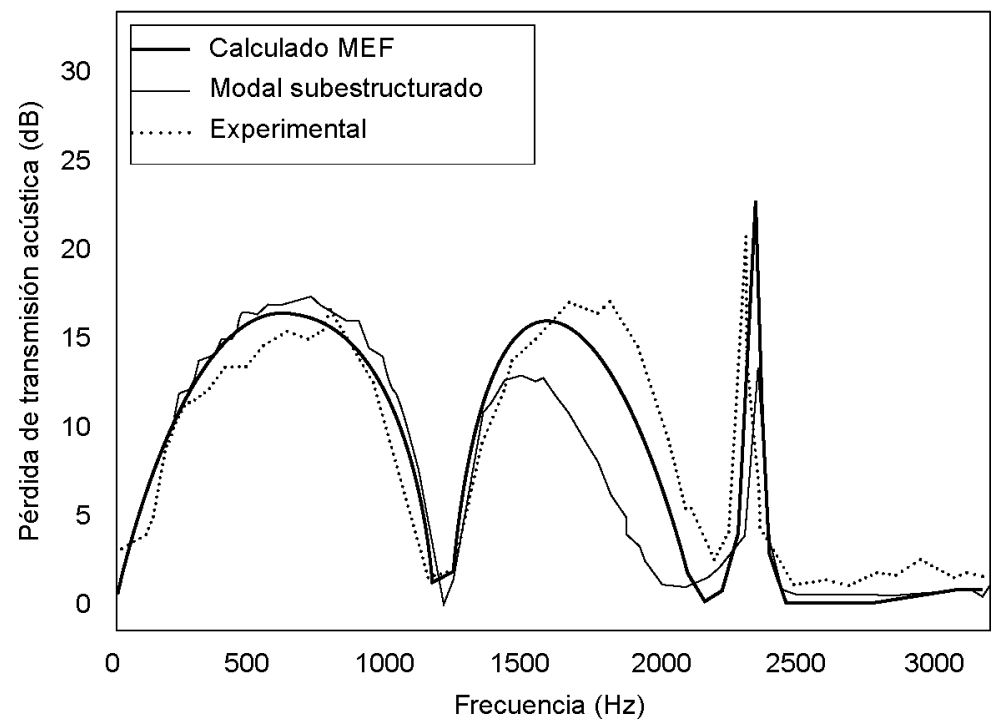

Figura 6. Resultado de TL para el SCP, validación de resultado.

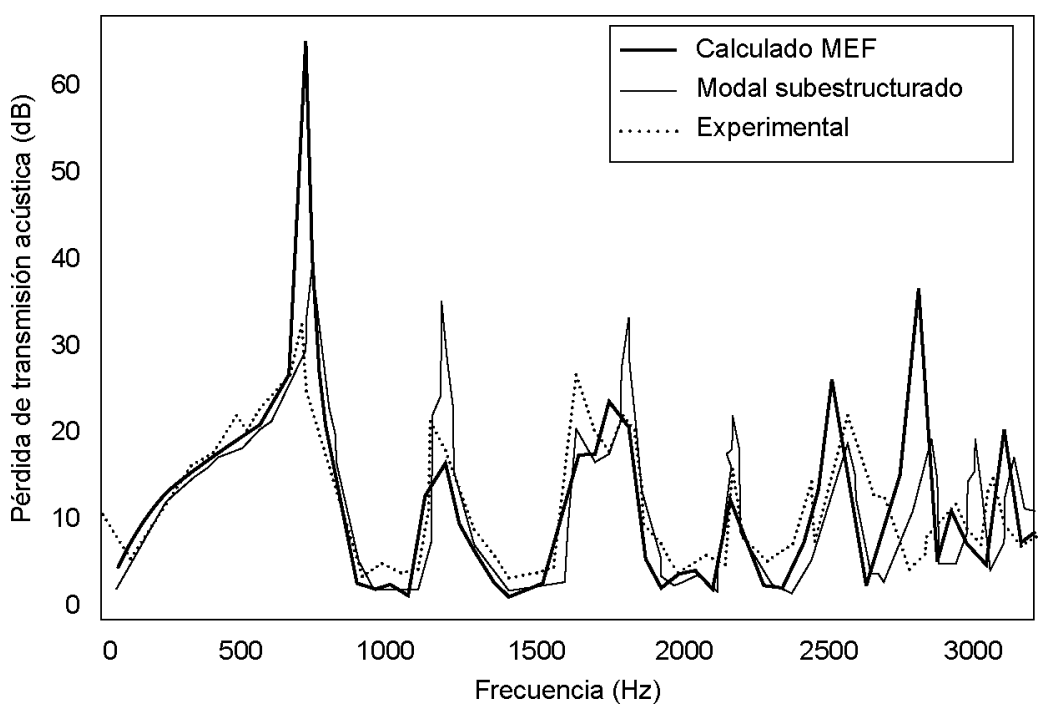

Figura 7. Resultado de TL para el SEP, validación de resultados. 
La calidad de los resultados obtenidos con la simulación en ANSYS, respecto a lo encontrado en la revisión bibliográfica, se realizó mediante el cálculo del error absoluto $\left(\varepsilon_{a}\right)$, (ecuación 8) y la diferencia euclidiana $\left(d_{E}\right)$ (ecuación 9). El procedimiento se realizó para los dos métodos, MEF y modal subestructurado.

$$
\begin{gathered}
\boldsymbol{\varepsilon}_{a}=\bar{X}-X_{i} \\
d_{E}\left(Q_{1}-Q_{2}\right)=\sqrt{\left(x_{2}-x_{1}\right)^{2}+\left(y_{2}-y_{1}\right)^{2}}
\end{gathered}
$$

Tabla 4. Error absoluto entre MEF y método modal subestructurado

\begin{tabular}{ccc}
\hline Tipo de silenciador & MEF error absoluto promedio (dB) & $\begin{array}{c}\text { Subestructurado error absoluto } \\
\text { promedio (dB) }\end{array}$ \\
\hline SCP & 1.6192 & 3.2099 \\
SPE & 4.2704 & 3.9218 \\
\hline
\end{tabular}

Tabla 5. Diferencia euclidiana entre MEF y método modal subestructurado

\begin{tabular}{ccc}
\hline Tipo de silenciador & FEM diferencia euclidiana (dB) & $\begin{array}{c}\text { Subestructurado diferencia } \\
\text { euclidiana (dB) }\end{array}$ \\
\hline SCP & 16.5058 & 43.2865 \\
SPE & 62.1055 & 42.2066 \\
\hline
\end{tabular}

En la tabla 6 se presentan los datos de caída de presión obtenidos con NX Flow para los casos en estudio. Es posible observar que hay una similitud en la caída de presión encontrada para los dos casos estudiados, que tienen valores bajos si se comparan con otros tipos de silenciadores como los presentados por Méndez y Avella (2017). Es importante mencionar que, al haber menor caída de presión, el rendimiento y la eficiencia del motor no se verán afectados.

Tabla 6. Cálculo de la caída de presión

\begin{tabular}{lcccc}
\hline $\begin{array}{c}\text { Tipo de } \\
\text { Silenciador }\end{array}$ & $\begin{array}{c}\text { Presión de entrada } \\
\left(\frac{N}{m^{2}}\right)\end{array}$ & $\begin{array}{c}\text { Presión de salida } \\
\left(\frac{N}{m^{2}}\right)\end{array}$ & $\begin{array}{c}\text { Caída de presión } \\
\left(\frac{N}{m^{2}}\right)\end{array}$ & $\begin{array}{c}\text { Cantidad de } \\
\text { elementos }\end{array}$ \\
\hline SCP & 2.814 & 0.008 & 2.806 & 335359 \\
SEP & 2.886 & 0.01 & 2.876 & 413019 \\
\hline
\end{tabular}

Como análisis final, se compararon los dos casos planteados en relación a las dos variables simuladas en este documento (caída de presión y pérdida de transmisión acústica). Como se muestra en la tabla 7, en este caso se podría seleccionar el silenciador SEP, pues logra una mejor atenuación del ruido a pesar de tener una caída de presión mayor a la del SCP, pues la diferencia se da en la segunda cifra decimal. 
Tabla 7. Comparación de resultados entre los silenciadores SCP y SEP.

\begin{tabular}{lll}
\hline Tipo de Silenciador & $\begin{array}{c}\text { Pérdida de transmisión } \\
\text { acústica }(\mathbf{d B}) \mathbf{a} 600 \mathrm{~Hz}\end{array}$ & $\begin{array}{c}\text { Caída de presión } \\
\left(\frac{N}{m^{2}}\right)\end{array}$ \\
\hline SCP & 16.4348 & 2.806 \\
SEP & 23.7052 & 2.876 \\
\hline
\end{tabular}

\section{Conclusiones}

En las figuras 6 y 7 se observa la capacidad del MEF para aproximarse a los valores experimentales. La diferencia entre los valores calculados y los presentados por Torres (2006), tanto a nivel numérico, como experimental, puede estar relacionada con la falta de planos de detalle de los casos simulados.

En la tabla 6 se observa como la combinación de una alta porosidad junto con una cámara ovalada y excéntrica (Tipo SEP) se convierte en una excelente base para desarrollar un silenciador más completo, que generaría una mayor atenuación acústica y una baja caída de presión. Al añadir cámaras y ductos perforados en una sola configuración, se puede crear un silenciador en el que la onda acústica recorra más obstáculos, de manera que se pierda mayor cantidad de energía, con lo que se lograría que los gases de escape provenientes del motor presenten menos resistencia de salir del sistema de escape.

En términos generales, los resultados obtenidos corresponden con lo mencionado en la sección introductoria, lo que sugiere que el $\mathrm{MEF}$ tiene alta capacidad para aproximar el comportamiento de un silenciador. Cabe resaltar que en este trabajo las condiciones de frontera se impusieron acorde con lo encontrado en la revisión bibliográfica, para así lograr la validación de los resultados obtenidos mediante la simulación planteada en este artículo.

\section{REFERENCIAS}

Ansys Inc. (2017). FLUID30 3-D Acoustic Fluid. Recueprado de http://www.ansys.stuba.sk/ html/elem_55/chapter4/ES4-30.htm

Cui, Z., y Huang, Y. (2016). Boundary element analysis of muffler transmission loss with LSDYNA. Ponencia presentada en 2th International LS-DYNA ${ }^{\circledR}$ Users Conference, DYNAmore y Livermore Software Technology Corp, Dearborn, Michigan, EE. UU.

García, A. (2014). Estudio comparativo del desempeño de los métodos FEM y MMT para el cálculo de la atenuación sonora de silenciadores pasivos (tesis de maestría). Maestría en Ingeniería, Universidad Nacional de Colombia, Bogotá, Colombia.

Martínez, M. (2010). Modelado analítico-numérico y caracterización experimental de silenciadores de escape hibridos (tesis de doctorado). Departamento de Ingeniería Mecánica y de Materiales, Universitat Politècnica de València, Valencia, España.

McMahon, K. (2014). A comparison of the transfer matrix method and the finite element method for the calculation of the transmission loss in a single expansion chamber muffler (tesis de maestría). Master of Engineering, Rensselaer Polytechnic Institute, New York, EE. UU.

Méndez, M., y Avella, M. (2017). Simulación de la caida de presión y de las condiciones acuisticas en un sistema de escape convencionalk (tesis de pregrado). Programa de Ingeniería Mecánica, Fundación Universidad de América, Bogotá, Colombia. 
Mishra, P., Kar, S., Mishra, H., y Gupta, A. (2016). Modeling for combined effect of muffler geometry modification and blended fuel use on exhaust performance of a four stroke engine: A computational fluid dynamics approach. Applied Thermal Engineering, 108, 1105-1118. https://doi.org/10.1016/j.applthermaleng.2016.08.009

Mori, G. (2016). Análisis estructural y atenuante de un silenciador (trabajo de grado). Departamento de Ingeniería Mecánica y Fabricación, Universidad de Sevilla, Sevilla, España.

Nefske, D., Wolf, J., y Howell, L. (1982). Structural-acoustic finite element analysis of the automobile passenger compartment: a review of current practice. Journal of Sound and Vibration, 80(2), 247-266.. https://doi.org/10.1016/0022-460X(82)90194-8

Pal, S. (2015). Design and acoustic analysis of exhaust muffers for automotive applications (tesis). M. Tech Machine Design, Departmento de Mechanical Engineering, Christ University, Bangalore, India.

Resolución 0627 de 2006. Por la cual se establece la norma nacional de emisión de ruido y ruido ambiental. Diario Oficial 46239 de abril 12 de 2006

Selamet, A., Denia, F., y Besa, A. (2003). Acoustic behavior of circular dual-chamber mufflers. Journal of Sound and Vibration, 265, 967-985. https://doi.org/10.1016/S0022-460X(02)01258-0

Siemens. (2013). NX Flow [software]. www.siemens.com/nx

Torres, M. (2006, March 31). Modelado acústico de silenciadores con material absorbente (tesis doctoral). Ingeniería Mecánica, Universitat Politècnica de València, Valencia, España. Recuperado de https://doi.org/10.4995/Thesis/10251/1892

Vergara, P. (2015). Implementación de plataforma de monitoreo zabbix para sistemas de telecomunicaciones Telsur (tesis de pregrado). Facultad de Ciencias de la Ingeniería, Universidad Austral de Chile, Valdivia, Chile. Recuperada de http://cybertesis.uach.cl/tesis/uach/2014/bmfciu.41i/ doc/bmfciu.41i.pdf

Yasuda, T., Wu, C., Nakagawa, N., y Nagamura, K. (2013). Studies on an automobile muffler with the acoustic characteristic of low-pass filter and Helmholtz resonator. Applied Acoustics, 74(1), 49-57. https://doi.org/10.1016/j.apacoust.2012.06.007 